\title{
On the force-velocity relationship of a bundle of rigid bio-filaments
}

Alessia Perilli, Carlo Pierleoni, Giovanni Ciccotti, and Jean-Paul Ryckaert

Citation: The Journal of Chemical Physics 148, 095101 (2018); doi: 10.1063/1.5001124

View online: https://doi.org/10.1063/1.5001124

View Table of Contents: http://aip.scitation.org/toc/jcp/148/9

Published by the American Institute of Physics

\section{Articles you may be interested in}

Relaxation rates of gene expression kinetics reveal the feedback signs of autoregulatory gene networks The Journal of Chemical Physics 148, 095102 (2018); 10.1063/1.5009749

Anomalies in the coil-stretch transition of flexible polymers

The Journal of Chemical Physics 148, 094903 (2018); 10.1063/1.5017555

Role of translational entropy in spatially inhomogeneous, coarse-grained models

The Journal of Chemical Physics 148, 094112 (2018); 10.1063/1.5018178

Proteins at the air-water interface in a lattice model

The Journal of Chemical Physics 148, 094704 (2018); 10.1063/1.5017789

Hydration dynamics of a lipid membrane: Hydrogen bond networks and lipid-lipid associations

The Journal of Chemical Physics 148, 094901 (2018); 10.1063/1.5011803

Force-extension behavior of DNA in the presence of DNA-bending nucleoid associated proteins

The Journal of Chemical Physics 148, 084902 (2018); 10.1063/1.5016177

\section{AIP $\left.\right|^{\text {The Journal of }}$ Chemical Physics}




\title{
On the force-velocity relationship of a bundle of rigid bio-filaments
}

\author{
Alessia Perilli, ${ }^{1, a)}$ Carlo Pierleoni, ${ }^{2, b)}$ Giovanni Ciccotti, ${ }^{3, c)}$ and Jean-Paul Ryckaert ${ }^{4, d)}$ \\ ${ }^{1}$ Department of Physics, Sapienza University of Rome, P.le Aldo Moro 5, I-00185 Rome, Italy and Department \\ of Chemistry, École Normale Supérieure, Rue Lhomond 24, 75005 Paris, France \\ ${ }^{2}$ Department of Physical and Chemical Sciences, University of L'Aquila, Via Vetoio 10, 67100 L'Aquila, \\ Italy and Maison de la Simulation, CEA, CNRS, Université Paris-Sud, UVSQ, Université Paris-Saclay, \\ 91191 Gif-sur-Yvette, France \\ ${ }^{3}$ Instituto per le Applicazioni del Calcolo "Mauro Picone" (IAC), CNR, Via dei Taurini 19, I-00185 Rome, Italy; \\ Sapienza University of Rome, P.le Aldo Moro 5, I-00185 Rome, Italy; and University College Dublin (UCD), \\ Belfield Dublin 4, Ireland \\ ${ }^{4}$ Department of Physics, Université Libre de Brussels (ULB), Campus Plaine, CP 223, B-1050 Brussels, Belgium
}

(Received 22 August 2017; accepted 10 February 2018; published online 1 March 2018)

\begin{abstract}
In various cellular processes, bio-filaments like F-actin and F-tubulin are able to exploit chemical energy associated with polymerization to perform mechanical work against an obstacle loaded with an external force. The force-velocity relationship quantitatively summarizes the nature of this process. By a stochastic dynamical model, we give, together with the evolution of a staggered bundle of $N_{f}$ rigid living filaments facing a loaded wall, the corresponding force-velocity relationship. We compute the evolution of the model in the infinite wall diffusion limit and in supercritical conditions (monomer density reduced by critical density $\hat{\rho}_{1}>1$ ), and we show that this solution remains valid for moderate non-zero values of the ratio between the wall diffusion and the chemical time scales. We consider two classical protocols: the bundle is opposed either to a constant load or to an optical trap setup, characterized by a harmonic restoring force. The constant load case leads, for each $F$ value, to a stationary velocity $V^{\text {stat }}\left(F ; N_{f}, \hat{\rho}_{1}\right)$ after a relaxation with characteristic time $\tau_{\text {micro }}(F)$. When the bundle (initially taken as an assembly of filament seeds) is subjected to a harmonic restoring force (optical trap load), the bundle elongates and the load increases up to stalling over a characteristic time $\tau^{O T}$. Extracted from this single experiment, the force-velocity $V^{O T}\left(F ; N_{f}, \hat{\rho}_{1}\right)$ curve is found to coincide with $V^{\text {stat }}\left(F ; N_{f}, \hat{\rho}_{1}\right)$, except at low loads. We show that this result follows from the adiabatic separation between $\tau_{\text {micro }}$ and $\tau^{O T}$, i.e., $\tau_{\text {micro }} \ll \tau^{O T}$. Published by AIP Publishing. https://doi.org/10.1063/1.5001124
\end{abstract}

\section{INTRODUCTION}

Cell motility in vivo is a large-scale manifestation of the living character of the cytoskeleton bio-filament network. ${ }^{1}$ In particular, F-actin filaments produce growing lamellipodium or filopodium structures where G-actin monomers polymerize at the barbed end of the filaments, directly in contact with the cytoplasmic membrane. The speed of the membrane deformation/displacement at the leading edge of the cell adjusts itself so that the force generated by the growing filaments compensates the resisting load coming from the membrane tension. For living filaments opposing a loaded mobile obstacle, the macroscopic force-velocity relationship, $V(F)$, linking the obstacle velocity $V$ to the instantaneous applied load $F$ only, quantitatively summarizes the combined action of the elementary self-assembling processes. In such adiabatic conditions, implying a time scale separation between the self-assembling process and the response of the obstacle, the $V(F)$ dependence could be probed equivalently by different protocols like, to cite

\footnotetext{
a)alessia.perilli@roma1.infn.it

b)carlo.pierleoni@aquila.infn.it

c)giovanni.ciccotti@roma1.infn.it

d)jryckaer@ulb.ac.be
}

the two most frequently used, the constant force load (e.g., clamped force setup), where one directly observes the steady state velocity, and the harmonic load (the sample grows against an AFM cantilever or an optical trap), where the obstacle velocity can be followed as the load increases continuously up to stalling.

Adiabatic conditions cannot be in general guaranteed and indeed careful investigations on a branched actin network growing against an AFM tip have shown that the recorded $V(F)$ relationship can be a function of the load history. ${ }^{2}$ The direct force-velocity relationship, $V(F)$, is in any event widely used as a characteristic of network dynamics to compare experimental measurements and modeling approaches for in vitro ${ }^{3}$ and in vivo systems. ${ }^{4}$

To make progress on the rationalization of the conditions of validity of the widely used concept of force-velocity relationship, $V(F)$, we will restrict our considerations to a simple network where a bundle of parallel filaments (actin or tubulin) grows normally against a loaded obstacle. Moreover, in order to keep the model as simple as possible, we will refer to experimental situations (in vitro experiments ${ }^{5,6}$ ) where flexibility of filaments or wall ATP (Adenosine Triphosphate) or GTP (Guanosine Triphosphate) hydrolysis, lateral interactions between filaments, and membrane attachment 
of filaments have negligible effects. In this way, we will concentrate on cases where the dynamic coupling between (de)polymerizing steps dominates the form of the forcevelocity relationship.

The general mechanism linking work production and (de)polymerization kinetics of living bio-filaments has been originally formulated by Hill for an incompressible bundle of $N_{f}$ parallel filaments pressing against a mobile obstacle. ${ }^{7}$ Successively, when the filaments of the bundle are treated as independent and equivalent and when it is assumed that the depolymerization rate is unaffected by the external load, the wall velocity $V^{M F}$ ( $M F$ indicates the mean field character of this treatment) has been written as ${ }^{8,9}$

$$
V^{M F}\left(F ; \rho_{1}, N_{f}\right)=d\left[U_{0} \exp \left(-\frac{F d}{N_{f} k_{B} T}\right)-W_{0}\right],
$$

where $U_{0}=k_{\text {on }} \rho_{1}$ and $W_{0}=k_{\text {off }}$ are the single filament bulk rate constants, related to bulk chemical rate constants $k_{\text {on }}$ and $k_{\text {off }}$, for single monomer polymerization and depolymerization steps, $\rho_{1}$ is the free monomer density, $d$ is the single filament increment of contour length per incorporated monomer, and $F$ is the external force exerted on the wall. Supercritical conditions, where filament polymerization dominates over depolymerization, require $\hat{\rho}_{1}=\frac{\rho_{1}}{\rho_{1 c}}=\frac{U_{0}}{W_{0}}>1$, where $\rho_{1 c}=\frac{k_{o f f}}{k_{o n}}$ is the critical value of the monomer density at which the bundle has no tendency to grow nor to shrink in the absence of load.

Equation (1) predicts, for $F=0$, a growth velocity of the free bundle $V^{M F}=d\left(U_{0}-W_{0}\right)>0$, while the stalling force $F_{s}$, at which the velocity vanishes, is given by

$$
F_{s}^{H}=N_{f} \frac{k_{B} T}{d} \ln \hat{\rho}_{1} .
$$

The notation $F_{s}^{H}$ reminds that this expression was originally established by Hill using thermodynamic arguments. ${ }^{10}$ Equation (2) has been recently derived, in the limit $d / L_{E Q} \rightarrow 0$, by equilibrium statistical mechanics for a bundle of rigid filaments. $^{11}$

Experimental measurements of the force-velocity rela-

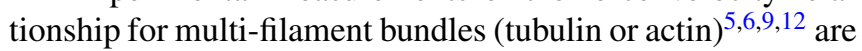
not many, reflecting the difficulty to prepare in vitro the grafted bundle seed needed to follow its subsequent loaded growth. However, it is interesting to note the diversity in these few approaches. The growth of single grafted tubulin filaments, which are bundles of 13 proto-filaments, was followed by imaging techniques. ${ }^{9,12}$ Regrouping $(F, V)$ data for different observation times and for different samples, a master forcevelocity relation could be established. In another experiment using an acrosome bead complex of $N_{f}=8 / 10$ F-actin filaments held in an optical trap device, the growth of a bundle was followed in time against a harmonic load. ${ }^{6}$ A rising signal finishing with a plateau was observed, but the final stationary force was surprisingly much lower than the expected stalling force, Eq. (2), its value being close to the stalling force predicted for a single filament. The analysis in this experiment considers many relaxation curves, but in many cases data had to be eliminated due to interferences during the relaxation process with the onset of escaping filaments. This happens because growing filaments undergo a large bending fluctuation which allows them to start growing freely along the obstacle. The transient behavior, which can be converted into a $V(F)$ law by estimating the time derivative of the wall position from the data, was not exploited. Finally, in a recent study, ${ }^{5}$ by recording the rate of the radial distance between two colloidal particles separated by a growing grafted actin bundle, the force-velocity relationship of actin bundles was established in constant load conditions.

The outcome of the earliest experiment, ${ }^{9,12}$ confirmed by the more recent experimental work, ${ }^{5}$ is that the velocity, and hence the power of transduction of multi-filament bundles, is much lower than that predicted by Eq. (1). The discrepancy highlights the correlation among elementary chemical steps at the tip of different filaments in the bundle, with the effect of reducing the additivity of the action of each filament. The bundle model needs to be specified and the dependence between chemical events and the wall position for a given longitudinal seed disposition has to be quantitatively taken into account. This aspect is present in the multi-filament Brownian Ratchet (BR) models ${ }^{5,9,13,14}$ which generalize the single filament Brownian ratchet model introduced by Peskin et al., ${ }^{15}$ for which one finds that the velocity vanishes for a load equal to Hill's expression, Eq. (2). ${ }^{9}$ For these bundle models, the important characteristics that distinguish the dynamical behavior of the bundle are the number of filaments $N_{f}$, the longitudinal disposition of the seeds of the filaments, and the wall diffusion coefficient $D$ that introduces a second characteristic time $\tau_{D}=d^{2} / D$ next to the chemical events' time scale $\tau_{\text {chem }}=W_{0}^{-1}$. This fact suggests us to introduce the parameter $\epsilon=\tau_{D} / \tau_{\text {chem }}$ to be able to discuss the condition of this second adiabatic separation [not to be confused with the one associated with the existence of $V(F)]$. For both experiments having probed the $V(F)$ relationship, it was found that data could be interpreted successfully with the model of a staggered bundle (=staggered longitudinal seed disposition ${ }^{11}$ ) of rigid filaments in infinite wall diffusion conditions $(\epsilon=0)$, a model we will denote as SRBR (Staggered Rigid Brownian Ratchet). On the contrary, for a similar model with an in registry bundle (unstaggered longitudinal seed disposition), ${ }^{14}$ the predicted velocity was much too low with respect to the experimental data. $5,9,12$

In the stochastic dynamical models considered here, the force-velocity relationship depends parametrically, for a given seed arrangement, on the number of filaments, the reduced free monomer concentration, and the time scale ratio $\epsilon$. In the case of constant load, the explicit form for the asymptotic force-velocity relationship, $V^{\text {stat }}\left(F ; N_{f}, \hat{\rho}_{1}, \epsilon\right)$, for our models has been established by stochastic dynamics studies at finite $\epsilon^{16}$ and at $\epsilon=0 .^{5,9}$ In the latter case, a simplified algorithm, exploiting the time scale separation, has been used for the staggered bundle case. Indeed, at $\epsilon=0$, the wall position distribution at a given filament configuration, is found to be time-independent and equal to the equilibrium distribution of the wall position resulting from the 1D Brownian motion of a wall in the external load field, with the wall positions restricted to be greater than the position of the most advanced filament tip.

Interestingly, a good approximation for the velocity $V^{\text {stat }}\left(F ; N_{f}, \hat{\rho}_{1}\right)$ and the distribution of filament relative sizes 
(see Sec. III) in the stationary state have been derived from the SRBR model.

In this work, we consider the stochastic staggered bundle model of rigid filaments in supercritical conditions and perform a series of dynamical runs for different load conditions. We first look at the constant force case, treating both the stationary state itself and the asymptotic transient evolution to reach it. We next envisage the bundle, in similar thermodynamic conditions, subject to a harmonic load $-\kappa_{T} L$, where $L$ is the wall position and where $\kappa_{T}$ is the trap strength (optical trap setup). Mimicking the optical trap experiment, ${ }^{6}$ the bundle, initially taken with very short filaments, and the average wall position grow and reach stalling. We compare our computed longest relaxation time with a theoretical approximate expression derived along the lines of Démoulin et al.'s theory. We derive and compare the force-velocity relationship extracted from this optical trap relaxation with the one obtained in stationary conditions. As expected, we found that the two coincide in adiabatic conditions, i.e., when the characteristic time of the optical trap relaxation is much larger than the characteristic time of the relaxation in the constant force case.

Our algorithms follow the same lines of those used in previous studies. However, in our study, we deal with an optical trap load, while most studies (with an exception restricted to the $\epsilon=0$ case $^{17}$ ) assume a constant load. Moreover, while algorithms for finite $\epsilon$ or $\epsilon=0$ are usually just assumed, we establish an explicit link showing how the $\epsilon=0$ model is derived from the general finite $\epsilon$ case.

In Sec. II, we present the general Fokker-Planck (FP) model for a bundle of rigid filaments with an arbitrary seed disposition, facing either a constant or a harmonic load, and we derive the explicit wall algorithm (EWA) giving the sampling rules to generate stochastic trajectories for any finite $\epsilon$ case. We then use a perturbation expansion to derive the $\epsilon=0$ model, still for the constant load or the harmonic load, and we derive the simplified implicit wall algorithm (IWA) giving the sampling rules in the $\epsilon=0$ case. Section III reports and discusses our results for constant force and optical trap loads for the same bundle system generally using the $\epsilon=0$ approach since the wall diffusion takes place very quickly with respect to the mean time between (de)polymerization events. However, we also verify that the simplified algorithm is robust since we find identical results in a reasonable range of $\epsilon$ non-zero values. Section IV concludes with a summary of the main results and with some perspectives.

\section{MODEL AND IMPLEMENTATION}

We consider a bundle of $N_{f} \geqslant 1$ living filaments, grafted normally (say along the $x$ axis) to a fixed planar substrate wall (along $y$ and $z$ directions). The filaments are modeled as discrete rigid linear chains with monomer contour incremental size $d$ and length related to the number of attached monomers, $j \geq 2$, as $L_{c j}=(j-1) d$. Let $h_{n}$ be the location along the $x$ axis of the seed (first monomer) of the filament $n$ close to the grafting plane $\left(-d / 2<h_{n}<d / 2\right)$. For a bundle of many filaments, two seed dispositions are usually considered: in-registry (or unstaggered), where $h_{n}=0, n=1,2, \ldots, N_{f}$, and homogeneous (or staggered), where seeds are regularly spaced as

$$
h_{n}=\left[\frac{n-0.5}{N_{f}}-0.5\right] d, \quad n=1,2, \ldots, N_{f},
$$

as illustrated for the $N_{f}=8$ case in Fig. 1 .

A moving obstacle, a hard wall located at distance $L$ from the parallel substrate wall, is loaded with a compressional external force $F$ bringing it into contact with the living filaments (see Fig. 1). We will consider two types of load, the constant force $F$ and the optical trap setting, with $F=-\kappa_{T} L$, where $\kappa_{T}$ is the trap stiffness and $L$ is the distance between the walls.

The bundle force for rigid filaments is impulsive. Its effect is taken into account by imposing a confining boundary to the wall motion at the tip location of the longest filament.

Filaments either grow by a single monomer polymerization step with bulk rate $U_{0}$, proportional to the free monomer density $\rho_{1}$, or shrink by a single monomer depolymerization step with bulk rate $W_{0}$. The ratio $U_{0} / W_{0}=\hat{\rho}_{1}$ is the free monomer density divided by its critical value, i.e., the value at which the two bulk rates are equal. We will be interested to supercritical conditions only $\left(\hat{\rho}_{1}>1\right)$, where the filaments tend to grow against the loaded wall. When a filament tip gets closer than $d$ to the wall, the polymerization rate becomes zero, while the depolymerization one is assumed to remain unchanged.

The dynamics of this simple model for a bundle of growing filaments against the loaded mobile wall present two main time scales: the chemical one, $\tau_{\text {chem }}=1 / W_{0} \sim 1 / U_{0}$, and the diffusive one, related to the diffusive motion of the wall and estimated by $\tau_{D}=d^{2} / D$ where $D$ is the wall diffusion coefficient. The ratio of time scales, $\epsilon=\tau_{D} / \tau_{\text {chem }}$, in typical in vitro experiments is $\epsilon \ll 1$ but might sometimes go close to 1 for a very large colloidal particle in a crowded environment.

In the following, we establish a Fokker-Planck equation to describe the dynamics of an arbitrary bundle of independent

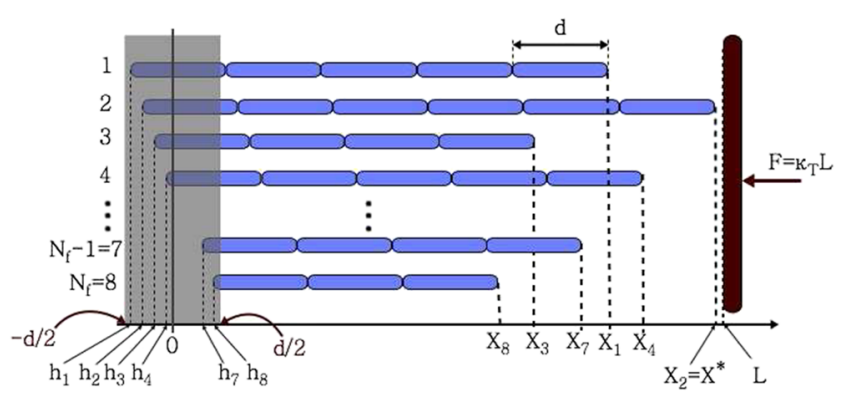

FIG. 1. Sketch of a homogeneous bundle of $N_{f}=8$ rigid filaments with monomer incremental contour length $d$ facing a rigid mobile wall located at $L$, subject to a harmonic force $F=-\kappa_{T} L$. The gray zone on the left highlights the thickness of the fixed wall at $x=0$ in which filaments are grafted, i.e., with their first monomer at $h_{n}$ [see Eq. (3)]. Given the filament tip positions $X_{n}$ for $n=1,2, \ldots, N_{f}$ [see Eqs. (4) and (5)], the most advanced filament with the tip located at $X^{*}$ corresponds to $n=2$ for the configuration shown. The loaded wall Brownian motion is thus restricted to a semi-infinite axis $L \geq X^{*}$ until a filament (de)polymerization step modifies the most advanced tip position. 
rigid filaments subjected to a constant or harmonic load, for an arbitrary value of $\epsilon$.

\section{A. General Fokker-Plank equation for a bundle of rigid filaments against a constant or harmonic load}

We describe the time evolution of $N_{f}$ filaments against a load in terms of the filament sizes and the wall position, $\left\{j_{1}, \ldots, j_{N_{f}}, L\right\}$. The wall position must always lie beyond the tip of any filament - and so beyond the tip of the most advanced one, $n^{*}$ with size $j_{n^{*}}$. We denote $X_{n}\left(j_{n}\right)$ the position of the tip of filament $n$ (composed by $j_{n}$ monomers) and $X^{*}$ the position of the most advanced tip:

$$
\begin{aligned}
X_{n}\left(j_{n}\right) & =\left(j_{n}-1\right) d+h_{n}, \\
X^{*} \equiv X^{*}\left(j_{1}, \ldots, j_{N_{f}}\right) & =\max _{n=1,2, \ldots, N_{f}}\left\{X_{n}\left(j_{n}\right)\right\}=X_{n^{*}}\left(j_{n^{*}}\right) .
\end{aligned}
$$

Since the filaments' tips can't cross the wall, we have the condition

$$
L \geqslant X^{*}
$$

We assume that the joint probability distribution function $P_{j_{1}, \ldots, j_{N_{f}}}(L, t)$ satisfies a Fokker-Planck equation in time mixing a continuous process in space for the wall position with a discrete process for the filament sizes. For the model described above, we have

$$
\begin{aligned}
& \frac{\partial P_{j_{1}, \ldots, j_{N_{f}}}(L, t)}{\partial t}+\frac{\partial}{\partial L} J_{j_{1}, \ldots, j_{N_{f}}}(L, t) \\
& =U_{0}\left[\sum_{n=1}^{N_{f}}\left(1-\delta_{2, j_{n}}\right) \Theta\left(L-X_{n}\left(j_{n}\right)\right) P_{j_{1}, \ldots, j_{n}-1, \ldots, j_{N_{f}}}(L, t)-\sum_{n=1}^{N_{f}} \Theta\left(L-X_{n}\left(j_{n}+1\right)\right) P_{j_{1}, \ldots, j_{n}, \ldots, j_{N_{f}}}(L, t)\right] \\
& \quad+W_{0}\left[\sum_{n=1}^{N_{f}} P_{j_{1}, \ldots, j_{n}+1, \ldots, j_{N_{f}}}(L, t)-\sum_{n=1}^{N_{f}}\left(1-\delta_{2, j_{n}}\right) P_{j_{1}, \ldots, j_{n}, \ldots, j_{N_{f}}}(L, t)\right]
\end{aligned}
$$

where $\Theta(x)$ is the Heaviside step function and the probability current density is

$J_{j_{1}, \ldots, j_{N_{f}}}(L, t)=-D\left[\frac{\partial P_{j_{1}, \ldots, j_{N_{f}}}(L, t)}{\partial L}-\frac{F(L)}{k_{B} T} P_{j_{1}, \ldots, j_{N_{f}}}(L, t)\right]$.

In Eq. (8), the compressive force can be either a constant $F<0$ or an elastic force $F(L)=-\kappa_{T} L$ modeling the optical trap. The right-hand side of Eq. (7) represents the sink and source terms affecting the dynamics due to polymerization and depolymerization events. Their explicit expression indicates that, in one step at fixed $L$, transitions are only possible between adjacent microscopic states, where $\left(N_{f}-1\right)$ filaments have the same size while the size of the remaining filament differs by \pm 1 unit, taking into account the restriction $L \geqslant X^{*}(t)$, and that the filament size cannot be smaller than two, $\left(1-\delta_{2, j_{n}}\right)$.

The general normalization condition for the distribution $P_{j_{1}, \ldots, j_{N_{f}}}(L, t)$ is

$$
\sum_{j_{1}=2}^{\infty} \cdots \sum_{j_{N_{f}}=2}^{\infty} \int_{X^{*}(t)}^{\infty} d L P_{j_{1}, \ldots, j_{N_{f}}}(L, t)=1
$$

while the boundary conditions on the probabilities are

$$
\begin{array}{cc}
\left.P_{j_{1}, \ldots, j_{N_{f}}}(L, t)\right|_{L<X^{*}(t)}=0, & \left.P_{j_{1}, \ldots, j_{N_{f}}}(L, t)\right|_{L=\infty}=0, \\
\left.J_{j_{1}, \ldots, j_{N_{f}}}(L, t)\right|_{L=X^{*}(t)}=0, & \left.J_{j_{1}, \ldots, j_{N_{f}}}(L, t)\right|_{L=\infty}=0 .
\end{array}
$$

To simplify the treatment of the continuous-discrete structure of Eq. (7), we discretize the wall position with a grid step $\delta=d / M$, with $M$ integer and $M \gg 1$, following Ref. 18 . We then substitute to the wall position $L$ the discrete variable

$$
k=\operatorname{int}\left[\frac{L}{\delta}\right] \equiv \text { int }[l] .
$$

In this way, Eq. (7) will become a finite difference equation in $k$ representing a discrete Markov chain in continuous time,

$$
\frac{d \mathcal{P}}{d t}=\mathcal{P} \mathcal{Q}
$$

with $\mathcal{P}(t)=\left\{\mathcal{P}_{j_{1}, \ldots, j_{N_{f}}, k}(t)\right\}_{j_{n} \in[2, \infty)} n=1,2, \ldots, N_{f}, k \in\left[\operatorname{int}\left[\left(d+h_{N_{f}}\right) / \delta\right], \infty\right)$ being a vector field and $\mathcal{Q}$ being the generator matrix of the Markov chain. The elements of the matrix $\mathcal{Q}$ contain the (de)polymerization rates for the filaments,

$$
\begin{aligned}
U_{j_{n}}(L) & =U_{0} \Theta\left(L-X_{n}\left(j_{n}+1\right)\right), \\
W_{j_{n}}(L) & =W_{0},
\end{aligned}
$$

and the forward/backward jump rates for the wall; the expressions of these matrix elements are given in Appendix A.

To circumvent the difficulty of solving analytically Eq. (13), one can produce a number of realizations of the discrete Markov chain using any appropriate algorithm, in our case the Gillespie algorithm: ${ }^{19,20}$ given an initial condition at time $t_{0}$, the state of the system is estimated in terms of the set of random variables $\left\{j_{1}, \ldots, j_{N_{f}}, k\right\}$ at time $t$ producing statistically correct trajectories, from which the probability distribution function $\mathcal{P}_{j_{1}, \ldots, j_{N_{f}}, k}(t)$ can be inferred by histograms. Starting from the initial state, the system is allowed to evolve by random steps involving only one event per time: one filament depolymerization or polymerization, or the wall forward or backward jump. Denoting by $i_{0}$ the current state of the system, the reachable states $i_{m}$ are those differing from $i_{0}$ for 
only one variable by \pm 1 , namely, $\left\{j_{1}, \ldots, j_{n} \pm 1, \ldots, j_{N_{f}}, k\right\}$ or $\left\{j_{1}, \ldots, j_{n}, \ldots, j_{N_{f}}, k \pm 1\right\}$. It is straightforward to see that the number of these possible final states is $2 N_{f}+2$. The transitions $i_{0} \rightarrow i_{m}, m \in\left[1,2 N_{f}+2\right]$, are described in Eq. (13) by the generator matrix elements $Q_{i_{m} i_{0}}$, the rates of going from $i_{0}$ to $i_{m}$. The corresponding diagonal element is $Q_{i_{0} i_{0}}$ $=-\sum_{i_{m} \neq i_{0}} Q_{i_{m} i_{0}} .{ }^{21}$ The evolution of the system is determined by two random variables: the time to the next reaction, $\tau$, and the final state $i_{m}$, or equivalently the index of the reaction, $m \in\left[1,2 N_{f}+2\right]$. From general Markov chain theory, $\tau$ is known to be an exponentially distributed random variable: given the current state $i_{0}$, the parameter of the exponential distribution is given by $-Q_{i_{0} i_{0}}$. Instead, the probability for the jump $m$ linking states $i_{0}$ and $i_{m}$ to take place is given by the ratio between $Q_{i_{0} i_{m}}$ and $\left|Q_{i_{0} i_{0}}\right|{ }^{21}$ The main loop of the algorithm follows this scheme:

0 . The initial state $i_{0}$ is specified in terms of the state vector $\left\{j_{1}, \ldots, j_{N_{f}}, k\right\}$. We take for the initial value of $k$ a small fixed value and, for the filament, compatible initial sizes.

1. The matrix elements $Q_{i_{0} i_{m}}$ are calculated for any state $i_{m}$ reachable from $i_{0}$.

2. The time to the next move is determined using the socalled direct method, which follows from the standard inversion method of the Monte Carlo theory: ${ }^{22}$ a random number $r_{1} \in[0,1]$ is generated from the uniform distribution and the time $\tau$ is taken as

$$
\tau=\frac{1}{\left|Q_{i_{0} i_{0}}\right|} \ln \frac{1}{r_{1}} .
$$

3. The index of the next move is determined using the same method: a second random number $r_{2} \in[0,1]$ is generated and the index $m$ is taken as the smallest integer satisfying

$$
\sum_{n=1}^{m-1} \frac{Q_{i_{0} i_{n}}}{\left|Q_{i_{0} i_{0}}\right|}<r_{2} \leqslant \sum_{n=1}^{m} \frac{Q_{i_{0} i_{n}}}{\left|Q_{i_{0} i_{0}}\right|} .
$$

4. The sampled move is taken by updating the state vector $i_{0} \rightarrow i_{m}$ and the time is incremented by $\tau$.

5. Go back to 1 , until a maximum time $t_{\max }$ is reached.

6. End the simulation.

The state vector $\left\{j_{1}, \ldots, j_{N_{f}}, k\right\}$ is stored for the calculation of histograms and averages.

This algorithm, ${ }^{18,19}$ solving the Fokker-Planck equations (7) and (8), works for any seed disposition (staggered and unstaggered), for any finite value of the dimensionless parameter $\epsilon \equiv \frac{d^{2} W_{0}}{D}$, and for both the two cases of constant force and optical trap load. We will call it the explicit wall algorithm (EWA).

In Subsection II B, we treat the specific, important reference case of loaded bundles of rigid filaments in the limit $\epsilon \rightarrow 0$. In this limit, the wall re-equilibrates instantaneously after any change of the position of the most advanced tip of the bundle. The interest of this limit is justified since in in vitro experiment with actin bundles/colloidal particles (e.g., the optical trap experiment ${ }^{6}$ ), the typical value of the ratio of time scales is $\epsilon \ll 1$. We will see that the dynamics of the bundle simplify for two reasons: the elimination of the fast motion of the wall permits to go to longer times and the dimensionality of the problem is reduced. The new algorithm, called the implicit wall algorithm (IWA), then becomes decidedly more efficient.

\section{B. Treatment of the Fokker-Planck equation in the $\epsilon=0$ limit}

Given the separation of time scales between the chemical events and the wall diffusion, it is convenient to rewrite ${ }^{23}$ Eq. (7) in terms of dimensionless variables in order to put in evidence the ratio $\epsilon=\frac{\tau_{D}}{\tau_{\text {chem }}}=\frac{W_{0} d^{2}}{D}$. Defining $\tilde{t}=W_{0} t, x=\frac{L}{d}$, and $f=\frac{F d}{k_{B} T}$, multiplying Eq. (7) by $\frac{d^{2}}{D}$, and redefining the probability distribution functions, we get

$$
\begin{aligned}
\epsilon & \frac{\partial \widetilde{P}_{j_{1}, \ldots, j_{N_{f}}}(x, \tilde{t})}{\partial \tilde{t}}+\frac{\partial}{\partial x} \widetilde{J}_{j_{1}, \ldots, j_{N_{f}}}(x, \tilde{t}) \\
= & \epsilon\left\{\hat{\rho}_{1}\left[\sum_{n=1}^{N_{f}}\left(1-\delta_{2, j_{n}}\right) \Theta\left(x-\frac{X_{n}\left(j_{n}\right)}{d}\right) \widetilde{P}_{j_{1}, \ldots, j_{n}-1, \ldots, j_{N_{f}}}(x, \tilde{t})-\sum_{n=1}^{N_{f}} \Theta\left(x-\frac{X_{n}\left(j_{n}+1\right)}{d}\right) \widetilde{P}_{j_{1}, \ldots, j_{n}, \ldots, j_{N_{f}}}(x, \tilde{t})\right]\right. \\
& \left.+\sum_{n=1}^{N_{f}} \widetilde{P}_{j_{1}, \ldots, j_{n}+1, \ldots, j_{N_{f}}}(x, \tilde{t})-\sum_{n=1}^{N_{f}}\left(1-\delta_{2, j_{n}}\right) \widetilde{P}_{j_{1}, \ldots, j_{n}, \ldots, j_{N_{f}}}(x, \tilde{t})\right\}
\end{aligned}
$$

with

$$
\widetilde{J}_{j_{1}, \ldots, j_{N_{f}}}(x, \tilde{t})=-\frac{\partial}{\partial x} \widetilde{P}_{j_{1}, \ldots, j_{N_{f}}}(x, \tilde{t})-f(x) \widetilde{P}_{j_{1}, \ldots, j_{N_{f}}}(x, \tilde{t})
$$

being the probability current density in the reduced units. In the $\epsilon \rightarrow 0$ limit, it is legitimate to replace Eq. (18) by its simpler $\epsilon$ zero-th order approximation

$$
\frac{\partial^{2} \widetilde{P}_{j_{1}, \ldots, j_{N_{f}}}^{(0)}(x, \tilde{t})}{\partial x^{2}}-\frac{\partial}{\partial x}\left[-f(x) \widetilde{P}_{j_{1}, \ldots, j_{N_{f}}}^{(0)}(x, \tilde{t})\right]=0 .
$$

By integrating in $d x$ from $X^{*}$ to $\infty$ and using the boundary conditions for the probability, one gets $\frac{\partial \widetilde{P}^{(0)}}{\partial x}=-f(x) \widetilde{P}^{(0)}$ so that the general solution is

$$
\widetilde{P}_{j_{1}, \ldots, j_{N_{f}}}^{(0)}(x, \tilde{t})=a\left(j_{1}, \ldots, j_{N_{f}}, \tilde{t}\right) \exp \left(-\int_{x}^{\infty} d x f(x)\right) .
$$


On the other hand, it is always possible to write the joint probability as the product of the marginal distribution for the subset $\left\{j_{1}, \ldots, j_{N_{f}}\right\}$ times the conditional probability distribution for $x$,

$$
\widetilde{P}_{j_{1}, \ldots, j_{N_{f}}}^{(0)}(x, \tilde{t})=\widetilde{P}_{0}\left(j_{1}, \ldots, j_{N_{f}}, \tilde{t}\right) \widetilde{P}_{0}\left(x \mid j_{1}, \ldots, j_{N_{f}}, \tilde{t}\right) .
$$

Therefore, given the general solution (21), we can write it as

$$
\widetilde{P}_{j_{1}, \ldots, j_{N_{f}}}^{(0)}(x, \tilde{t})=\widetilde{P}_{0}\left(j_{1}, \ldots, j_{N_{f}}, \tilde{t}\right) \widetilde{P}_{E Q}\left(x \mid j_{1}, \ldots, j_{N_{f}}\right)
$$

as the $x$ dependence, Eq. (21), is explicit and time-independent. The wall distribution $\widetilde{P}_{E Q}\left(x \mid j_{1}, \ldots, j_{N_{f}}\right)$ is an explicit, timeindependent, and normalized distribution for the wall position conditional to the set of filament sizes. Explicit expression for the two normalized cases of constant load and optical trap is

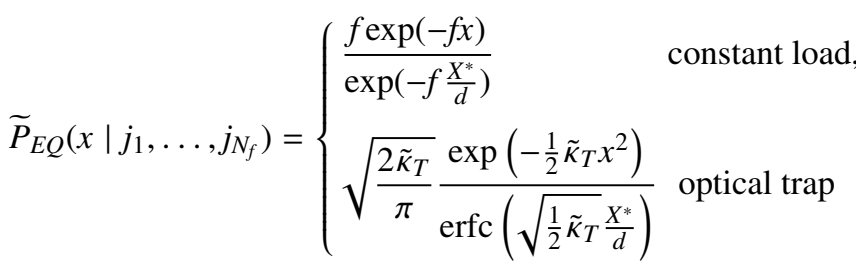

with $\tilde{\kappa}_{T}=\frac{\kappa_{T} d^{2}}{k_{B} T}$. From Eq. (24), we get the average wall position conditional to the bundle sizes $\left\{j_{1}, \ldots, j_{N_{f}}\right\}$ as

$$
\begin{aligned}
E\left(x \mid j_{1}, \ldots, j_{N_{f}}\right)= & \int_{X^{*}}^{\infty} x \widetilde{P}_{E Q}\left(x \mid j_{1}, \ldots, j_{N_{f}}\right) d x \\
& = \begin{cases}\frac{X^{*}}{d}+\frac{1}{f} \quad \text { constant load, } \\
\sqrt{\frac{2}{\tilde{\kappa}_{T} \pi} \frac{\exp \left[-\frac{1}{2} \tilde{\kappa}_{T}\left(\frac{X^{*}}{d}\right)^{2}\right]}{\operatorname{erfc}\left(\sqrt{\frac{1}{2} \tilde{\kappa}_{T}} \frac{X^{*}}{d}\right)}} \text { optical trap. }\end{cases}
\end{aligned}
$$

Note that the full distribution at $\epsilon=0$, given by Eq. (23), is still a time-dependent function since filament sizes change by single monomer polymerization/depolymerization events; the infinite separation of the time scales $(\epsilon=0)$ implies that after any chemical event, the wall immediately re-equilibrates according to the time-independent distribution, Eq. (24), given the new set of filament sizes. To get the full distribution, we write $\widetilde{P}_{j_{1}, \ldots, j_{N_{f}}}(x, \tilde{t})$ as an asymptotic expansion in terms of the small parameter $\epsilon$,

$$
\widetilde{P}_{j_{1}, \ldots, j_{N_{f}}}(x, \tilde{t})=\widetilde{P}_{j_{1}, \ldots, j_{N_{f}}}^{(0)}(x, \tilde{t})+\epsilon \widetilde{P}_{j_{1}, \ldots, j_{N_{f}}}^{(1)}(x, \tilde{t})+\cdots,
$$

where $\widetilde{P}_{j_{1}, \ldots, j_{N_{f}}}^{(0)}(x, \tilde{t})$ is given by Eq. (23). If we substitute this expansion, truncated to the first order, into Eq. (18), to the order $\epsilon$, we find the following equation:

$$
\begin{aligned}
\frac{\partial \widetilde{P}_{j_{1}, \ldots, j_{N_{f}}}^{(0)}(x, \tilde{t})}{\partial \tilde{t}}+\frac{\partial}{\partial x} \widetilde{J}_{j_{1}, \ldots, j_{N_{f}}}^{(1)}(x, \tilde{t}) \\
=\hat{\rho}_{1}\left[\sum_{n=1}^{N_{f}}\left(1-\delta_{2, j_{n}}\right) \Theta\left(x-\frac{X_{n}\left(j_{n}\right)}{d}\right) \widetilde{P}_{j_{1}, \ldots, j_{n}-1, \ldots, j_{N_{f}}}^{(0)}(x, \tilde{t})-\sum_{n=1}^{N_{f}} \Theta\left(x-\frac{X_{n}\left(j_{n}+1\right)}{d}\right) \widetilde{P}_{j_{1}, \ldots, j_{n}, \ldots, j_{N_{f}}}^{(0)}(x, \tilde{t})\right] \\
\quad+\sum_{n=1}^{N_{f}} \widetilde{P}_{j_{1}, \ldots, j_{n}+1, \ldots, j_{N_{f}}}^{(0)}(x, \tilde{t})-\sum_{n=1}^{N_{f}}\left(1-\delta_{2, j_{n}}\right) \widetilde{P}_{j_{1}, \ldots, j_{n}, \ldots, j_{N_{f}}}^{(0)}(x, \tilde{t}) .
\end{aligned}
$$

Integrating both sides of this equation from $x=X^{*} / d$ to $\infty$, applying the boundary conditions Eq. (11) on $\widetilde{J}^{(1)}$ and the normalization of $\widetilde{P}_{E Q}$, and using Eq. (23), we get

$$
\begin{aligned}
& \frac{\partial \widetilde{P}_{0}\left(j_{1}, \ldots, j_{N_{f}}, \tilde{t}\right)}{\partial \tilde{t}} \\
& =\hat{\rho}_{1} \sum_{n=1}^{N_{f}}\left(1-\delta_{2, j_{n}}\right) \int_{\frac{X^{*}}{d}}^{\infty} d x \Theta\left(x-\frac{X_{n}\left(j_{n}\right)}{d}\right) \widetilde{P}_{E Q}\left(x \mid j_{1}, \ldots, j_{n}-1, \ldots, j_{N_{f}}\right) \widetilde{P}_{0}\left(j_{1}, \ldots, j_{n}-1, \ldots, j_{N_{f}}, \tilde{t}\right) \\
& \quad-\hat{\rho}_{1} \sum_{n=1}^{N_{f}} \int_{\frac{X^{*}}{d}}^{\infty} d x \Theta\left(x-\frac{X_{n}\left(j_{n}+1\right)}{d}\right) \widetilde{P}_{E Q}\left(x \mid j_{1}, \ldots, j_{n}, \ldots, j_{N_{f}}\right) \widetilde{P}_{0}\left(j_{1}, \ldots, j_{n}, \ldots, j_{N_{f}}, \tilde{t}\right) \\
& \quad+\sum_{n=1}^{N_{f}} \widetilde{P}_{0}\left(j_{1}, \ldots, j_{n}+1, \ldots, j_{N_{f}}, \tilde{t}\right)-\sum_{n=1}^{N_{f}}\left(1-\delta_{2, j_{n}}\right) \widetilde{P}_{0}\left(j_{1}, \ldots, j_{n}, \ldots, j_{N_{f}}, \tilde{t}\right) .
\end{aligned}
$$

This equation describes a discrete process for the filament sizes in continuous time, which can be rewritten in a vectorial form, similar to Eq. (13),

$$
\frac{d P_{0}}{d t}=P_{0} \mathcal{Q}^{(0)}
$$

with $\mathcal{Q}^{(0)}$ being the generator matrix of the process, whose elements are given in Appendix B.

The numerical solution of the Markov chain equation described by Eq. (29) follows exactly the same scheme described above for the general Fokker-Planck equation for $\epsilon>0$. 
As already mentioned, in this case, the algorithm is more efficient since it spans longer times (we have integrated out the fast variable) and it has to treat a reduced number of variables.

The solution of Eq. (29) and the conditional probability for the wall position Eq. (24) give the necessary information needed to compute all time-dependent ensemble averages, as, e.g., $\langle L\rangle_{t}$. Similar model and procedures have been used (i) for constant load option and in-registry ${ }^{14}$ or staggered $^{5,9,13}$ bundles and (ii) for optical trap and staggered bundles only. ${ }^{17}$

\section{SIMULATIONS AND RESULTS}

\section{A. Units, parameters, and stochastic runs}

In our simulations, length, time, and energy units are taken as $d, W_{0}^{-1}$, and $k_{B} T$, respectively. All quantities will be mentioned in reduced units based on the above three fundamental units. For actin $d=2.7 \mathrm{~nm}$; experimental information for $W_{0}$ gives $W_{0}=1.4 \mathrm{~s}^{-1}$; and at room temperature, $k_{B} T=4.14$ $\times 10^{-21} \mathrm{~J}$. We choose to perform our studies on a bundle of $N_{f}=32$ rigid filaments with a staggered disposition of seeds at a reduced density $\hat{\rho}_{1}=\frac{U_{0}}{W_{0}}=2.5$. With reference to a wall constituted by a bead of micron size in water opposing the actin bundles, ${ }^{5,6}$ experimental information gives for the adimensional parameter introduced in Sec. II, the value $\epsilon=5.5 \times 10^{-5}$. Given the small value of $\epsilon$, we performed the major part of our simulations in the $\epsilon=0$ limit with the IWA. However, we have considered of some interest to compare the results of the IWA with those of the EWA corresponding to a finite but small value of $\epsilon$. With the very small experimental value of $\epsilon$, the EWA would be highly inefficient since the computer time would be essentially spent to study the wall diffusion next to a bundle with quasi-fixed filament sizes. Since for the load-velocity relationship we need to sample both wall and filament sizes, we decided to adopt a value of epsilon thousand times bigger, $\epsilon=5 \times 10^{-2}$. This value, in fact, still permits to give a sufficient representation of the wall dynamics. Our EWA approach requires to discretize the space variable $L$ with elementary steps $\delta=d / M$. For $M$, we have adopted $M=100$. To compute the solution of our Fokker-Planck equation, both for $\epsilon=0$ (IWA) or $\epsilon>0$ (EWA), we need to fix initial configurations. Our choice for EWA has been to fix the wall location $L_{0}$ [i.e., $k_{0}=$ int $\left.\left(L_{0} / \delta\right)\right]$ and to sample the initial filament sizes for each trajectory of the stochastic dynamics according to the filament size equilibrium probability $P^{e q}\left(j_{1}, j_{2}, \ldots, j_{n}, \ldots, j_{N_{f}} ; L_{0}\right)$, conditional to the chosen wall location. ${ }^{11}$ For initiating IWA runs, the initial filament sizes must be arbitrarily chosen and the initial wall location then follows from its conditional distribution.

\section{B. Observables of interest}

\section{(1) Wall position}

The wall position $L$ is the quantity directly followed in time in real experiments and corresponds to the expected value of the random variable $\hat{L}$ over the solution of the FP equation, $\langle\hat{L}\rangle_{t}$. The calculation of this quantity is direct in the EWA case, while it has to be determined in the IWA case through the instantaneous size distribution of $j_{n}, n=1,32$, implying $\hat{L}$ values ahead of the tip of the most advanced filament at $X^{*}$, given by Eq. (5), using Eq. (25).

(2) Relative size (in number of monomers) of filaments with respect to the leading one.

In terms of the tip positions $X_{n}$ and $X^{*}$ defined by Eqs. (3)-(5), let us define the relative subset index $m=1$, $N_{f}-1$ given by

$$
\begin{gathered}
m(n)=\bmod \left(\frac{X^{*}-X_{n}}{d / N_{f}}, N_{f}\right)=\bmod \left(\left(j_{n^{*}}-j_{n}\right) N_{f}+n^{*}-n, N_{f}\right), \\
n=1,2, \ldots, N_{f}, n \neq n^{*} .
\end{gathered}
$$

This index represents in successive order the filament of order $m$, nearest neighbor of $n^{*}$, second neighbor of $n^{*}$, etc. Therefore it gives an intrinsic order to the vector representing the relative size of each filament. Then we can define, for each filament $n$, the quantity

$$
\begin{aligned}
k_{m} & =\operatorname{int}\left[\frac{X^{*}-X_{n(m)}}{d}\right] \\
& =\operatorname{int}\left[j_{n^{*}}-j_{n(m)}+\frac{n^{*}-n(m)}{N_{f}}\right], \quad m=1,2, \ldots, N_{f}-1 .
\end{aligned}
$$

Each component of this vector represents in discrete units of monomer size $d$ the relative distance from the most advanced tip of the first, second, etc., neighboring index.

This vector of relative sizes is interesting because its timedependent probability distribution reaches a stationary value in the case of the wall subjected to a constant load.

(3) Density of relative size of $N_{f}-1$ filaments with respect to the leading one

This quantity is defined by the microscopic observable

$$
\hat{g}(k)=\frac{1}{\left(N_{f}-1\right)} \sum_{m=1}^{N_{f}-1} \delta_{k, k_{m}}
$$

and corresponding $k$ average value $\hat{k}_{a v}=\sum_{k=0}^{\infty} k \hat{g}(k)$. At time $t$, the microscopic distribution will be $g(k, t)=\langle\hat{g}(k)\rangle_{t}$. Specifically, we will characterize the internal structure of the bundle either by $g(0, t)$, the average probability density that the filament tips lie at a distance smaller than $d$ from the tip of the most advanced filament, or by the average relative size $\left\langle\hat{k}_{a v}\right\rangle_{t}=\sum_{k=0}^{\infty} k g(k, t)$. We will denote by $g(k)$ and $k_{a v}$ the time-asymptotic values of $\langle\hat{g}(k)\rangle_{t}$ and $\left\langle\hat{k}_{a v}\right\rangle_{t}$ for constant load dynamics.

\section{Constant load}

We have computed the relaxation toward the stationary state for a homogeneous bundle of $N_{f}=32$ rigid living filaments at $\hat{\rho}_{1}=2.5$ pressing against a constant load $F$. We have chosen various values of $F$ in the range $0.05<F / F_{s}<1.25$ with $F_{s}$ being the stalling force, Eq. (2). For each load value, we have used the IWA to produce $10^{4}$ independent trajectories, starting at time 0 with all filament sizes set to the same value $\left[j_{n}(0)=500, n=1,32\right]$. We have chosen this value to avoid to fall at later times at the lower boundary $j_{n}=2$. That could happen when $F>F_{s}$ with negative average velocities. 


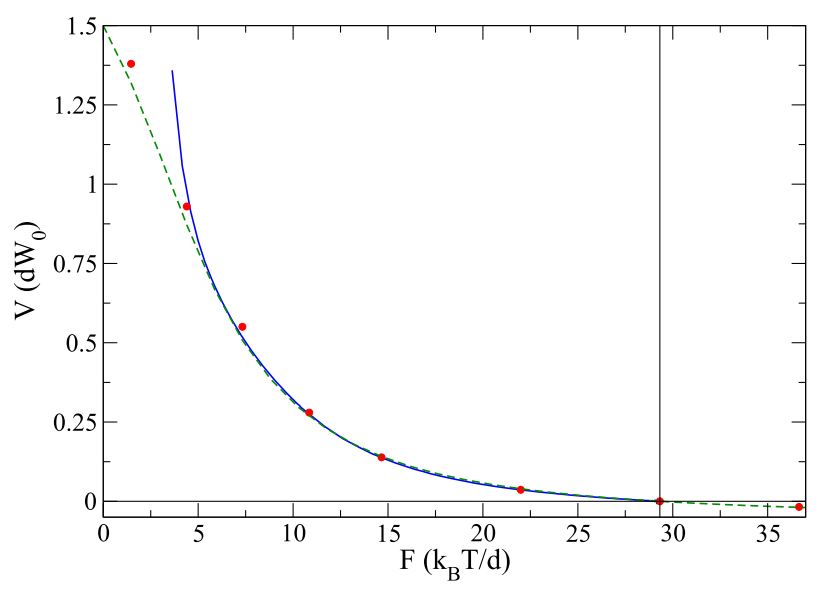

FIG. 2. Force-velocity relationship for a homogeneous bundle of $N_{f}=32$ rigid filaments at $\hat{\rho}_{1}=2.5(\epsilon=0)$. The $V^{\text {stat }}$ stationary velocity data points (red filled circles) are obtained as the asymptotic slope of $\langle\hat{L}\rangle_{t}$ for constant force runs at each shown load value. Error bars are less than symbol sizes. The dashed green line is Démoulin et al.'s theoretical estimate of $V^{\text {stat }}(F)$ based on Eqs. (C1) and (C2). The blue continuous curve is the force-velocity relationship obtained by the optical trap relaxation at $\kappa_{T}=0.4511$ (see text). Stalling is indicated by the vertical line at $F=29.32$.

In two cases, starting with $L_{0}=5 d$, we have used the EWA, averaging over $10^{3}$ independent trajectories. To determine the microscopic relaxation time of the bundle, we have fitted the asymptotic time evolution of the average wall position as $\langle L\rangle_{t}$ $=C+V^{\text {stat }} t+C^{\prime} \exp \left(-t / \tau_{\text {micro }}\right)$. To get the diffusion coefficient of the bundle $\Gamma$, we have also fitted the asymptotic behavior of the mean square elongation $\sigma^{2}(t)=\left\langle\hat{L}^{2}\right\rangle_{t}-\langle\hat{L}\rangle_{t}^{2} \underset{t \rightarrow \infty}{\sim} 2 \Gamma t .^{24}$

In Fig. 2, we report $V^{\text {stat }}(F)$ together with Démoulin et al.'s prediction [Eqs. (C1) and (C2)] for the staggered bundle of rigid filaments at $\epsilon=0$ in the same conditions. ${ }^{5}$ This comparison shows that the theoretical prediction of $V^{\text {stat }}(F)$ represents quite accurately (the difference never exceeding $2 \%$ ) the exact results obtained between zero load and stalling conditions.

In Fig. 3, we collect transient times $\tau_{\text {micro }}$ and the diffusion coefficient of the bundle, $\Gamma$. Note the consistency within $\Gamma$ values obtained from IWA or EWA runs. $\tau_{\text {micro }}$ results in the order of $W_{0}^{-1}$ except at small loads where it diverges; in Sec. IV, we will come back to this important point.

Figures 4 and 5 show, respectively, for the stationary state, the load-dependent averages $g(0 ; F)$ and $k_{a v}(F)$ Eqs. (31) and (32). Démoulin's predictions for the same quantities are also shown in these two figures, confirming their quantitative accuracy.

\section{Optical trap}

Let us start this section with an important remark: for our model, the choice of $\kappa_{T}$ appears to be completely arbitrary, although, of course, it should satisfy at least the condition that the final equilibrium value of the length of the bundle is much greater than $d,\langle\hat{L}\rangle_{E Q} / d \gg 1$, in order to avoid boundary effects. We will see below that the choice of a realistic $\kappa_{T}$ value (leading to $\langle\hat{L}\rangle_{E Q} / d \gg 1$ ) implies $\tau_{\text {micro }} \ll \tau_{O T}$ and hence it will guarantee the equivalence of the results of the optical trap setup against the constant load, at least for non-diverging $\tau_{\text {micro }}$.

Figure 6 shows time-dependent averages, $F_{t}=\kappa_{T}\langle\hat{L}\rangle_{t}$, for optical trap relaxations computed by the EWA and IWA for $\kappa_{T}=0.25$ and only by the IWA for $\kappa_{T}=0.4511$. In the EWA case with $\epsilon=0.05$, the relaxations start from a bundle size, short with respect to the final equilibrium value, i.e., $L_{0}=5 \mathrm{~d}$, while in the IWA case the filament sizes all start at $j_{n}=6$. The results, obtained by the two algorithms for $\kappa_{T}=0.25$, are indistinguishable, confirming the validity of the simplified algorithm. Note that the plateau values are in perfect agreement with the stalling force predicted by Hill, Eq. (2), within statistical error bars. Fluctuations of $\hat{L}$ at equilibrium is given, as expected, ${ }^{11}$ by $\sigma_{L}^{e q}=\sqrt{\left(k_{B} T / \kappa_{T}\right)}$. The vertical bars reported in the figure represent the standard deviation, $\kappa_{T} \sigma_{L}(t)$, associated with the fluctuation of the force. They remain bounded along the entire curve by the equilibrium value, indicating a limited fluctuation of the individual trajectories $\hat{L}(t)$. This is a relevant fact because experiments performed by an optical trap setup usually refer to a single trajectory measurement, ${ }^{6}$ whose representativeness of the ensemble average is guaranteed by the smallness of fluctuations.

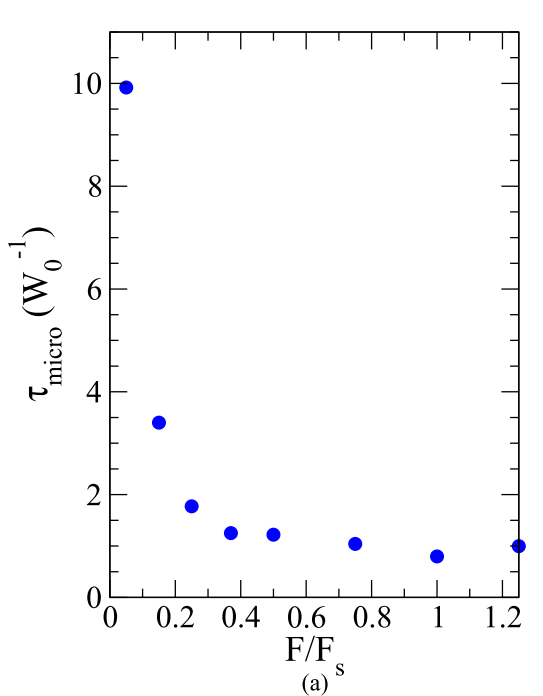

(a) $\mathrm{s}$

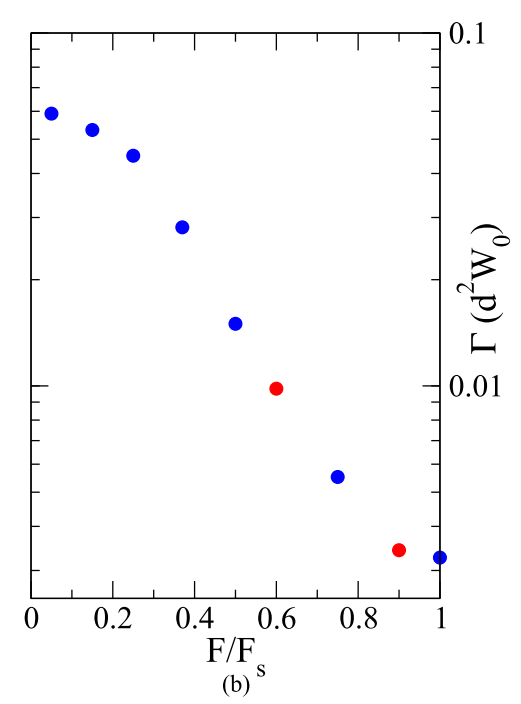

FIG. 3. (a) Load dependence of the relaxation time $\tau_{\text {micro }}$ for a homogeneous bundle of $N_{f}=32$ rigid filaments countering a constant load $F$ at $\hat{\rho}_{1}=2.5$. (b) Diffusion coefficient $\Gamma$ of the bundle. Blue symbols (IWA) and red symbols (EWA) refer to the stationary part of the constant load stochastic dynamics experiment mentioned in (a). 


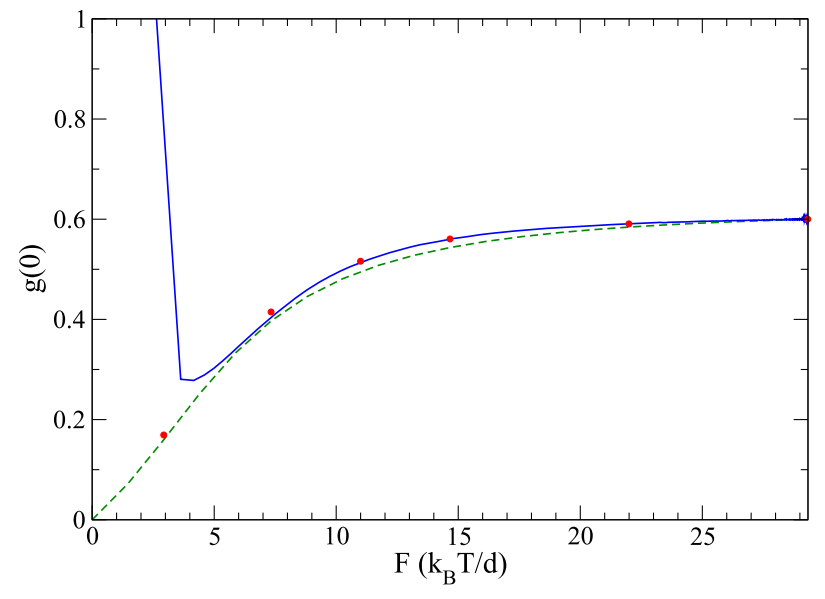

FIG. 4. Values at $k=0$ of the relative size distribution, $\mathrm{g}(0)$, as a function of the external load with $N_{f}=32, \hat{\rho}_{1}=2.5$, and $\epsilon=0$. The red filled circles are obtained in the stationary regime of constant load runs at the shown values of $F$. Error bars are less than symbol sizes. The dashed green line is Démoulin et al.'s estimate of $g(0 ; F)$ based on Eq. (C2). The blue continuous curve is obtained for the optical trap by eliminating from $g(0, t)=\langle\hat{g}(0)\rangle_{t}$ and $\langle F\rangle_{t}=\kappa_{T}\langle L\rangle_{t}$ at $\kappa_{T}=0.4511$, the time parameter $t$.

As Eq. (13) refers to a Markov process, one expects an asymptotic relaxation of $\langle L\rangle_{t}$ as

$$
\begin{aligned}
\langle L\rangle_{t} & =\langle L\rangle_{E Q}+A_{1} \exp \left(\lambda_{1} t\right)+\ldots \\
& =\langle L\rangle_{E Q}+A_{1} \exp \left(-\frac{t}{\tau}\right)+\cdots
\end{aligned}
$$

where $A_{1}$ is the amplitude (dependent on initial conditions) of the slowest, non-zero mode with eigenvalue $\lambda_{1}=-\frac{1}{\tau^{O T}}$ of the generator matrix governing the dynamics of the system. In the same long time limit, one has

$$
\begin{aligned}
& \langle F\rangle_{t}=\kappa_{T}\langle L\rangle_{t}=F_{s}+A_{1} \kappa_{T} \exp \left(\lambda_{1} t\right)+\cdots, \\
& \langle V\rangle_{t}=A_{1} \lambda_{1} \exp \left(\lambda_{1} t\right)+\cdots,
\end{aligned}
$$

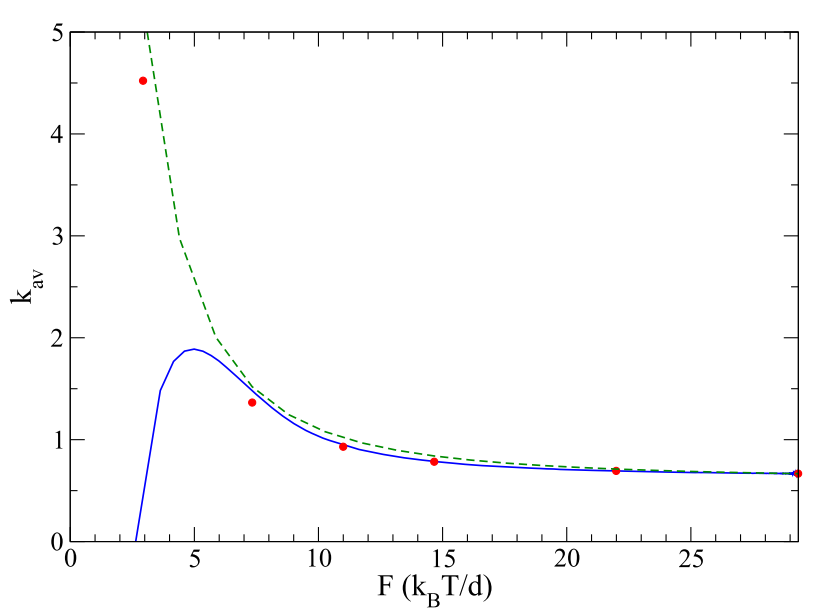

FIG. 5. Average filament relative size for $N_{f}=32$ at $\hat{\rho}_{1}=2.5$ and $\epsilon=0$. The red filled circles, denoting $k_{a v}$, are obtained in the stationary regime of constant load runs at each shown load value $F$. Error bars are less than symbol sizes. The dashed green line is Démoulin et al.'s theoretical estimate of $k_{a v}(F)$ based on Eq. (C4). The blue continuous curve is obtained for the optical trap setup by eliminating from $\left\langle\hat{k}_{a v}\right\rangle_{t}=\left\langle\sum_{k=0}^{\infty} k \hat{g}(k)\right\rangle_{t}$ and $\langle F\rangle_{t}=\kappa_{T}\langle L\rangle_{t}$ at $\kappa_{T}=0.4511$, the time parameter $t$.

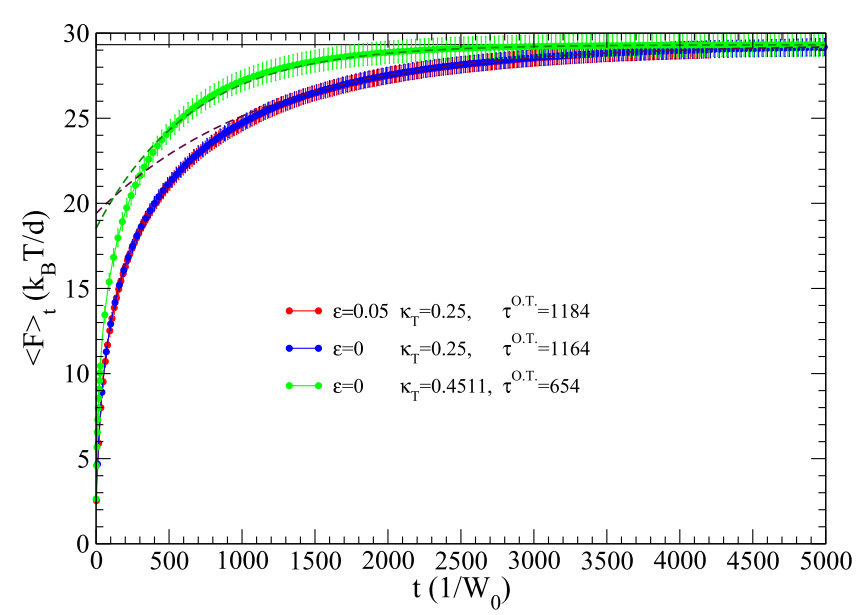

FIG. 6. Non-equilibrium relaxations of staggered bundles of $N_{f}=32$ rigid filaments growing in an optical trap at reduced density $\hat{\rho}_{1}=2.5$, all of them starting from initial conditions with the wall set to a value $L_{0} \approx 5 d$ $\ll\langle L\rangle_{E Q}$. The wall position $\langle L\rangle_{t}$ and the associated Root Mean Square Deviation (RMSD) $\sigma_{L}(t)$ are found as a function of time in the figure where what is effectively shown is the load evolution $\kappa_{T}\langle L\rangle_{t}$ and corresponding RMSD $\kappa_{T} \sigma_{L}(t)$. The final plateau value of the relaxations is compatible with the value $F_{s}=F_{s}^{H}$ given by Eq. (2) indicated by a horizontal thin black line. The dashed lines represent the best fit of an exponential asymptotic behavior Eq. (34), providing estimates of $\tau^{O T}$ and hence of the chemical friction $\gamma$ defined by Eq. (37). We find $\gamma=291 \pm 4$ (IWA with $\kappa_{T}=0.25$ ) and $\gamma=295 \pm 5$ (IWA with $\left.\kappa_{T}=0.4511\right)$ on the basis of the $\tau^{O T}$ values obtained.

and, thus, formally one can express the longest relaxation time of the optical trap relaxation as

$$
\tau^{O T}=-\lambda_{1}^{-1}=\kappa_{T}^{-1} \lim _{t \rightarrow \infty} \frac{F_{s}-\langle F\rangle_{t}}{\langle V\rangle_{t}} .
$$

From the data in Fig. 6 at $\kappa_{T}=0.25$, one gets $\tau^{O T}=1185$ \pm 50 for the EWA sampling, while the IWA sampling provides $\tau^{O T}=1164 \pm 10$. At $\kappa_{T}=0.4511$ for the IWA sampling, $\tau^{O T}=654 \pm 10$. By numerical differentiation, we have calculated the slopes of $\langle L\rangle_{t} \equiv l\left(t ; \kappa_{T}\right),\langle V\rangle_{t}=\frac{d\langle L\rangle_{t}}{d t} \equiv v\left(t ; \kappa_{T}\right)$. Eliminating $t$ from the pair of parametric equations $\left[\langle F\rangle_{t}\right.$ $\left.=\kappa_{T} l\left(t ; \kappa_{T}\right), v\left(t ; \kappa_{T}\right)\right]$, we can get the velocity as a function of the force, which is still a function of $\kappa_{T}$. The force-velocity relationship for $\kappa_{T}=0.4511$, shown in Fig. 2, turns out to be equivalent, except at small loads, to $V^{\text {stat }}(F)$ previously established for the constant force load stationary state. Identical results are obtained from the relaxation with $\kappa_{T}=0.25$ (not shown), indicating a negligible dependence, if any, of the force-velocity relationship on $\kappa_{T}$. The same agreement for $g(0)$ and $k_{a v}$ between the constant load results and the results obtained in optical trap is shown in Figs. 4 and 5, supporting the validity of the optical trap to extract the bundle behavior. Again for the present rigid model, the long time behavior is independent of the specific value of $\kappa_{T}$ adopted.

\section{E. Adiabaticity}

Figure 2 shows that, except at low forces (short time part of the relaxation behavior), $V^{\text {stat }}(F)$ superposes well the velocity-force relationship extracted from the optical trap relaxation. The identity between the stationary force-velocity relationship with the one obtained by the relaxation process in the optical trap setup is a clear indication of the fact that 
the optical trap setup is working in adiabatic conditions, i.e., we have a relaxation process happening in between stationary states. We can derive from this apparent adiabaticity, especially valid at long times in the optical trap relaxation when the load changes slowly in time, that

$$
\tau^{O T} \equiv \kappa_{T}^{-1} \lim _{t \rightarrow \infty} \frac{F_{s}-\langle F\rangle_{t}}{\langle V\rangle_{t}}=-\left[\kappa_{T}\left(\frac{\partial V^{s t a t}}{\partial F}\right)_{F_{s}}\right]^{-1} \equiv \frac{\gamma}{\kappa_{T}},
$$

where $V^{\text {stat }}(F)$ is the constant load force-velocity relationship and where $\gamma$, defined as minus the inverse of the slope of $V^{\text {stat }}(F)$ at stalling in Eq. (37), $\gamma=-\left[\left(\frac{\partial V^{\text {stat }}}{\partial F}\right)_{F_{s}}\right]^{-1}$, is a friction coefficient having a chemical (and not hydrodynamic) origin. The structure of the relaxation time expression Eq. (37) resembles that of an overdamped Brownian oscillator.

Equation (37) can be tested with our data. Using $\tau^{O T}$ estimates mentioned earlier for the two values of $\kappa_{T}$, we get three compatible $\gamma$ estimates $(291 \pm 4$ for the IWA run at $\kappa_{T}=0.25,296 \pm 12$ for EWA at $\kappa_{T}=0.25$, and $295 \pm 5$ for IWA at $\left.\kappa_{T}=0.4511\right)$. These values provide an overall estimate of $\gamma=293 \pm 3$ which has to be compared to the value of the slope of $V^{\text {stat }}(F)$ at stalling. The numerical derivative estimated with our wide spread data gives $\gamma=272$; unfortunately, this value is not sufficiently precise to be completely reliable. The uncertainty provided by computing the left and right incremental ratios giving, respectively, $\gamma=202$ and $\gamma=414$ tells us that we are within the numerical uncertainty. As for the Démoulin result, its approximate estimate of the slope leads to $\gamma^{\text {Dem }}=281.6$ (see Appendix C).

By referring the chemical friction coefficient $\gamma$ to the value characteristic of the mean field force-velocity relationship Eq. (2), $\gamma^{M F}=\frac{N_{f} k_{B} T}{d^{2} W_{0}}$, we can define a new adimensional coefficient, $C\left(N_{f}, \hat{\rho}_{1}\right)$, as

$$
C\left(N_{f}, \hat{\rho}_{1}\right)=\frac{\gamma}{\gamma^{M F}}=\frac{d^{2} W_{0}}{N_{f} k_{B} T} \gamma=9.2 \pm 0.1,
$$

giving a measure of the dynamic correlations between filaments. Note that $C=1$ not only in the MF model but also in the single filament Brownian ratchet in the $\epsilon=0$ limit because the force-velocity relationship is identical to the MF expression for $N_{f}=1$. The Démoulin estimate of $C$ gives in our case $\left(N_{f}=32, \hat{\rho}_{1}=2.5\right) C^{\text {Dem }}=8.8$.

On an intuitive basis, adiabaticity is related to a very fast equilibration of filament sizes during the slow (long time) nonequilibrium evolution of the optical trap, with respect to the microscopic relaxation time of the filaments under constant load, $\tau_{\text {micro }}$. The characteristic time of the optical trap equilibration is $\tau^{O T}$. We have seen, in Fig. 3, that, for $F / F_{s}>0.15$, the typical microscopic relaxation time $\tau_{\text {micro }}$ lies in the range $\approx(1-3) W_{0}^{-1}$. Now we can explain what we have anticipated at the beginning of this section: with the values we have chosen for $\kappa_{T}$, corresponding to equilibrium sizes of the bundle well satisfying the condition $L_{E Q} / d \gg 1$ (to avoid boundary problems associated to the short size of the bundle), the values of $\tau^{O T}$ result automatically to be two to three orders of magnitude larger (see the values given in Fig. 6). It is important to stress, however, that $\tau_{\text {micro }}$ values diverge as $F / F_{s} \rightarrow 0$, a property paralleled by the divergence in the same limit of $k_{a v}$.

\section{CONCLUDING REMARKS}

In this work, we have considered, in a Markovian approximation, a stochastic dynamical model to compute the evolution and the statistical properties of a staggered bundle of $N_{f}$ rigid living filaments growing against a loaded wall. In the FokkerPlanck equations, we have written down to give an explicit dynamics to our system, a parameter, $\epsilon=\tau_{D} / \tau_{\text {chem }}$, which plays a special role. Generally, the model has to be solved for the values of $\epsilon$ relatively small. It is found that if we take the $\epsilon$ $=0$ limit, the dynamics simplify and the overall computations become much lighter. We have shown numerically that the results obtained in a reasonable range of non-zero values of $\epsilon$ in the neighborhood of zero coincide with the results obtained using the limiting model and the simplified algorithm. This indicates the robustness of the $\epsilon=0$ limit. As a consequence, the major part of the computational work of the present paper has been performed in this limit. For the loading of the wall, we considered two classical protocols: a constant load or an optical trap setup, characterized by a harmonic restoring force. By a series of computer experiments at constant load and by a single calculation in the optical trap, we have obtained for the two protocols the classical force-velocity relationship. With the exception of the region of very weak loads, we have found the perfect coincidence of the results. We have been able to explain this universality of the response as a result of the time scale separation between the relaxation time needed by the wall to adjust to a change of the external force in the optical trap and the characteristic time needed by the chemistry to change the conformation of the bundle. This condition is violated when the load is very small (i.e., the trap expands rather fast) and the optical trap and constant load results differ, even dramatically, in that region. Our results suggest that experiments measuring the force-velocity relationship with a harmonic load offer, in principle, many advantages over the approach where constant force setups are used. Indeed, only a single sample is needed to get a $V(F)$ estimate over a large $F$ window in the first case, while a separate experiment and in general a specific sample are needed for each steady state at constant load $F$ investigated. Alternative protocols are possible, like imaging techniques used in Ref. 12, but the rules needed to get adiabaticity are easily transposed. We have been also able to confirm the validity of the approximate theory developed by Démoulin et al. ${ }^{5}$ to compute the properties of our system.

In this work, we have considered non-interacting rigid filaments subject to one polymerization rate and one depolymerization rate facing a hard mobile wall. Rigid filament models incorporating in addition lateral interactions (between protofilaments $)^{25}$ and hydrolysis effects ${ }^{26}$ or both $^{27}$ have been studied for actin and microtubules. For multi-filament bundles, they give rise to new collective effects affecting the load-velocity relationship and the stalling force. It would be interesting to test whether the adiabatic character of the optical trap response remains verified when these more realistic features of filaments are incorporated in the bundle model. 
Interpreting experimental data with rigid models implies that the semi-flexible character of living bio-filaments has limited influence on the results. How the bundle dynamics is affected by the flexibility is a delicate point, which is largely unknown, and this, to some extent, hampers the confidence in interpreting data with rigid filament models. Work is in progress to clarify the influence of flexibility on the force-velocity relation. ${ }^{28}$

\section{ACKNOWLEDGMENTS}

We thank G. Kozyreff for his help with the perturbation expansion of the FP equation in Sec. II B. Two of us (C.P. and J.P.R.) are grateful to J. Baudry, J. F. Joanny, and D. Lacoste for useful discussions. We thank G. Destrée for technical help. J.P.R. thanks the University of L'Aquila for hospitality during a three-month visit. C.P. is supported by the Agency Nationale de la Recherche (ANR) under the project "HyLightExtreme."

\section{APPENDIX A: DISCRETIZED FOKKER-PLANCK EQUATION FOR THE WALL-BUNDLE SYSTEM IN AN OPTICAL TRAP OR CONSTANT FORCE SETUP}

In this appendix, we derive a proper discretization of the Fokker-Planck equation together with the elements of the generator matrix $\mathcal{Q}$ of the Markov process given by Eq. (13).

To get the matrix elements that account for the discretization of the variable $L$, following the procedure introduced in Ref. 18, we concentrate only on the diffusive part of Eq. (7) for the wall position probability at given chemical state, $P_{j_{1}, \ldots, j_{N_{f}}}(L, t) \equiv P_{j}(L, t)$

$$
\frac{\partial P_{j}(L, t)}{\partial t}=-\frac{\partial}{\partial L} J_{j}(L, t),
$$

where $J_{j}(L, t)=-D\left(\frac{\partial P_{j}(L, t)}{\partial L}+\frac{1}{k_{B} T} \frac{d \Phi}{d L} P_{j}(L, t)\right)$ is the probability current, with $\frac{d \Phi}{d L}=\kappa_{T} L$ or $-F$ for, respectively, the optical trap or the constant force setup. We define the probabilities for the wall to be in the intervals $(l=L / \delta) k-1 / 2 \leqslant l<k+1 / 2$ and $k+1 / 2 \leqslant l<k+3 / 2$ as

$$
\begin{aligned}
p_{k}(t) & =\int_{k-1 / 2}^{k+1 / 2} P_{j}(l, t) d l, \\
p_{k+1}(t) & =\int_{k+1 / 2}^{k+3 / 2} P_{j}(l, t) d l .
\end{aligned}
$$

By defining the wall forward rate $F_{k+1 / 2}$ of going from $k$ to $k+1\left(F_{k-1 / 2}\right.$ from $k-1$ to $\left.k\right)$ and the wall backward rate $B_{k+1 / 2}$ of going from $k+1$ to $k\left(B_{k-1 / 2}\right.$ from $k$ to $\left.k-1\right)$, the time evolution of the probability $p_{k}(t)$ can be written as ${ }^{18}$

$$
\begin{aligned}
\frac{d p_{k}(t)}{d t} & =F_{k-1 / 2} p_{k-1}-\left(F_{k+1 / 2}+B_{k-1 / 2}\right) p_{k}+B_{k+1 / 2} p_{k+1} \\
& =-\left(F_{k+1 / 2} p_{k}-B_{k+1 / 2} p_{k+1}\right)+\left(F_{k-1 / 2} p_{k-1}-B_{k-1 / 2} p_{k}\right) \\
& =-\left(J_{k+1 / 2}-J_{k-1 / 2}\right)
\end{aligned}
$$

where the rates $F_{k \pm 1 / 2}$ and $B_{k \pm 1 / 2}$ have to be derived by discretizing Eq. (A1). $J_{k+1 / 2}$ is the net probability flux between sites $k$ and $k+1\left(J_{k-1 / 2}\right.$ is between $k-1$ and $\left.k\right)$.
If we now discretize Eq. (A1) using, e.g., the central difference method $\left[f_{k+1 / 2}^{\prime}=\left(f_{k+1}-f_{k}\right) / \delta\right]$ and compare the resulting discrete equation with Eq. (A4), the forward and backward rates obtained will not respect the detailed balance, a sufficient condition to reach equilibrium, while we expect the evolution of the Markov chain to lead to it, with each process balanced by its reverse.

To overcome this difficulty, following Ref. 18, we can look for the stationary solution of Eq. (A1) and see if, by integration over a proper interval of lengths, we can identify the rates, bringing us to coefficients satisfying the detailed balance.

Looking at the definitions Eqs. (A2) and (A3), we see that to get $p_{k}$ and $p_{k+1}$ from a solution of the stationary equation (A1), we need to solve it in the interval $(k-1 / 2, k+3 / 2)$. Then we look for the solution of the probability $P_{j}(L, t)$ in terms of the stationary solution $P_{E Q}(l)$ of

$$
D \frac{d}{d l}\left(\frac{d P_{E Q}(l)}{d l}+\frac{\Delta \Phi_{k+1 / 2}}{k_{B} T} P_{E Q}(l)\right)=0
$$

in $l \in(k-1 / 2, k+3 / 2)$, where we have substituted to $d \Phi / d l$ by the constant approximation $\Delta \Phi_{k+1 / 2}$, with

$$
\Delta \Phi_{k+1 / 2}=\Phi(k+1)-\Phi(k)
$$

The general solution of Eq. (A5) is $P_{E Q}(l)=\eta \exp \left(-\frac{\Delta \Phi_{k+1 / 2}}{k_{B} T} l\right)$ $+\theta$ with $\eta$ and $\theta$ constants. Plugging this expression into Eqs. (A2) and (A3), one can easily find $\eta$ and $\theta$ in terms of $p_{k}$ and $p_{k+1}$. Then the (approximate) stationary solution of the Fokker-Planck equation for the wall in the interval $(k-1 / 2$, $k+3 / 2)$ is

$$
\begin{aligned}
P_{E Q}(l)= & \frac{\Delta \Phi_{k+1 / 2}\left(p_{k}-p_{k+1}\right)}{k_{B} T\left(\exp \left(-\frac{\Delta \Phi_{k+1 / 2}}{k_{B} T}\right)-1\right)^{2}} \exp \left(\frac{\Delta \Phi_{k+1 / 2}}{k_{B} T}(k-1 / 2)\right) \\
& \times \exp \left(-\frac{\Delta \Phi_{k+1 / 2}}{k_{B} T} l\right)+\frac{p_{k} \exp \left(-\frac{\Delta \Phi_{k+1 / 2}}{k_{B} T}\right)-p_{k+1}}{\left(\exp \left(-\frac{\Delta \Phi_{k+1 / 2}}{k_{B} T}\right)-1\right)} \\
& \times l \in(k-1 / 2, k+3 / 2) .
\end{aligned}
$$

From this equation, we get the probability flux in the same interval

$$
\begin{aligned}
J_{E Q}(l) & =-\widetilde{D} \frac{d P_{E Q}(l)}{d l}-\widetilde{D} \frac{\Delta \Phi_{k+1 / 2}}{k_{B} T} P_{E Q}(l) \\
& =-\frac{\widetilde{D} \Delta \Phi_{k+1 / 2}}{k_{B} T} \frac{p_{k} \exp \left(-\frac{\Delta \Phi_{k+1 / 2}}{k_{B} T}\right)-p_{k+1}}{\left(\exp \left(-\frac{\Delta \Phi_{k+1 / 2}}{k_{B} T}\right)-1\right)}
\end{aligned}
$$

with $\widetilde{D}=D / \delta^{2}$ being the diffusion constant in $\delta$ units. Comparing this current with the probability flux defined in Eq. (A4), we get the following forward and backward rates:

$$
F_{k+1 / 2}=\widetilde{D} \frac{\Delta \Phi_{k+1 / 2} / k_{B} T}{\exp \left(\frac{\Delta \Phi_{k+1 / 2}}{k_{B} T}\right)-1}
$$




$$
B_{k+1 / 2}=\widetilde{D} \frac{-\Delta \Phi_{k+1 / 2} / k_{B} T}{\exp \left(-\frac{\Delta \Phi_{k+1 / 2}}{k_{B} T}\right)-1}
$$

The same approach for the interval $(k-3 / 2, k+1 / 2)$ can be used to get $F_{k-1 / 2}$ and $B_{k-1 / 2}$.

By direct substitution, we see that Eqs. (A9) and (A10) respect the detailed balance, $F_{k+1 / 2} P_{E Q}(k)=B_{k+1 / 2} P_{E Q}(k+1)$.
Substituting the appropriate expression for $\Phi\left(L_{k}\right)$, we have

$$
\Delta \Phi_{k+1 / 2}= \begin{cases}F \delta & \text { constant load, } \\ \frac{1}{2} \kappa_{T} \delta^{2}\left((k+1)^{2}-k^{2}\right) & \text { optical trap. }\end{cases}
$$

The non-zero elements of the generator matrix $\mathcal{Q}$ can now be written as follows:

$$
\begin{aligned}
& \mathcal{Q}_{\left\{j_{1}, \ldots, j_{n}, \ldots, j_{N_{f}}, k\right\}\left\{j_{1}, \ldots, j_{n}, \ldots, j_{N_{f}}, k+1\right\}}=F_{k+1 / 2}, \\
& \mathcal{Q}_{\left\{j_{1}, \ldots, j_{n}, \ldots, j_{N_{f}}, k\right\}\left\{j_{1}, \ldots, j_{n}, \ldots . j_{N_{f}}, k-1\right\}}=C_{k-1 / 2}= \begin{cases}B_{k-1 / 2} & \text { if } k-1 \geqslant X^{*} / d, \\
0 & \text { otherwise },\end{cases} \\
& \mathcal{Q}_{\left\{j_{1}, \ldots, j_{n}, \ldots, j_{N_{f}}, k\right\}\left\{j_{1}, \ldots, j_{n}+1, \ldots, j_{N_{f}}, k\right\}}=U_{j_{n}}=\left\{\begin{array}{cc}
U_{0} & \text { if } k \geqslant X_{n}\left(j_{n}+1\right) / d, \\
0 & \text { otherwise },
\end{array}\right. \\
& \mathcal{Q}_{\left\{j_{1}, \ldots, j_{n}, \ldots, j_{N_{f}}, k\right\}\left\{j_{1}, \ldots, j_{n}-1, \ldots, j_{N_{f}}, k\right\}}=W_{j_{n}}=W_{0}, \\
& \mathcal{Q}_{\left\{j_{1}, \ldots, j_{n}, \ldots, j_{N_{f}}, k\right\}\left\{j_{1}, \ldots . j_{n}, \ldots, j_{N_{f}}, k\right\}}=-F_{k+1 / 2}-C_{k-1 / 2}-\sum_{n=1}^{N_{f}}\left(U_{j_{n}}+W_{j_{n}}\right)
\end{aligned}
$$

with $X_{n}\left(j_{n}\right)$ and $X^{*}$ given by Eqs. (4) and (5). The row sums of this matrix are zero, as required for a generator matrix of a Markov chain,

$$
\sum_{\left\{j_{1}^{\prime}, \ldots, j_{n}^{\prime}, \ldots, j_{N_{f}}^{\prime}, k^{\prime}\right\}} \mathcal{Q}_{\left\{j_{1}, \ldots, j_{n}, \ldots, j_{N_{f}}, k\right\}\left\{j_{1}^{\prime}, \ldots, j_{n}^{\prime}, \ldots, j_{N_{f}}^{\prime}, k^{\prime}\right\}}=0 .
$$

Equation (13) represents hence a continuous time Markov process with discrete states; as for the variable $L$, the discrete states are approximations (exact in the $\delta \rightarrow 0$ limit) to the continuous/discrete process defined in Eq. (7).

\section{APPENDIX B: ELEMENTS OF THE $\epsilon=0$ GENERATOR MATRIX}

In this appendix, we write explicitly the matrix elements of $\mathcal{Q}^{(0)}$, generator of the Markov process in the $\epsilon=0$ limit Eq. (29). Since in this limit, the integration in $L$ allowed us to get rid of the continuous wall diffusion process, these elements can be written immediately,

$$
\begin{aligned}
\mathcal{Q}_{\left\{j_{1}, \ldots, j_{n}, \ldots, j_{N_{f}}\right\}\left\{j_{1}, \ldots, j_{n}+1, \ldots, j_{N_{f}}\right\}}^{(0)} & =U_{j_{n}} \\
& =U_{0} A^{(n)}\left(j_{1}, \ldots, j_{n}, \ldots, j_{N_{f}}\right), \\
\mathcal{Q}_{\left\{j_{1}, \ldots, j_{n}, \ldots, j_{j_{f}}\right\}\left\{j_{1}, \ldots, j_{n}-1, \ldots, j_{N_{f}}\right\}}^{(0)} & =W_{j_{n}}=W_{0}, \\
\mathcal{Q}_{\left.j_{1}, \ldots, j_{n}, \ldots, j_{N_{f}}\right\}\left\{j_{1}, \ldots, j_{n}, \ldots, j_{N_{f}}\right\}}^{(0)} & =-\sum_{n=1}^{N_{f}}\left(U_{j_{n}}+W_{j_{n}}\right),
\end{aligned}
$$

where $A^{(n)}\left(j_{1}, \ldots, j_{n}, \ldots, j_{N_{f}}\right)$ is given by

$$
\begin{aligned}
& A^{(n)}\left(j_{1}, \ldots, j_{n}, \ldots, j_{N_{f}}\right) \\
& \quad=\int_{X^{*} / d}^{\infty} d x \Theta\left(x-X_{n}\left(j_{n}+1\right) / d\right) \widetilde{P}_{E Q}\left(x \mid j_{1}, \ldots, j_{n}, \ldots, j_{N_{f}}\right) \\
& \quad= \begin{cases}\exp \left[-f\left(X^{*^{\prime}}-X^{*}\right) / d\right] & \text { constant load, } \\
\frac{\operatorname{erfc}\left[\left(\widetilde{\kappa}_{T} / 2\right)^{1 / 2} X^{*^{\prime}} / d\right]}{\operatorname{erfc}\left[\left(\widetilde{\kappa}_{T} / 2\right)^{1 / 2} X^{*} / d\right]} & \text { optical trap, }\end{cases}
\end{aligned}
$$

where $X^{*^{\prime}}$ is the most advanced filament's tip for the set of filament sizes $\left\{j_{1}, \ldots, j_{n}+1, \ldots, j_{N_{f}}\right\}$. Equation (B4) has been derived previously for constant load ${ }^{5,9}$ and for optical trap load. ${ }^{17}$

\section{APPENDIX C: DÉMOULIN ET AL.'S PREDICTION FOR $V(F)$ AND $k_{a v}$}

Démoulin et al. ${ }^{5}$ have proposed an approximate solution for the force-velocity relationship of staggered rigid filaments subjected to a constant load $F$ in the $\epsilon=0$ limit. They found that

$$
\begin{aligned}
V(F)= & \frac{d U_{0}}{N_{f}}\left[N_{f} \exp \left(-\frac{F d}{k_{B} T}\right)\right. \\
& \left.+\sum_{m=1}^{N_{f}-1} g(0)\left(N_{f}-m\right) \exp \left(-\frac{F d\left(N_{f}-m\right)}{N_{f} k_{B} T}\right)\right] \\
& -\frac{d W_{0}}{N_{f}}\left[g(0) \sum_{m=1}^{N_{f}-1} m(1-g(0))^{m-1}+N_{f}(1-g(0))^{N_{f}-1}\right]
\end{aligned}
$$

with the relative size distribution $g(k)$ given by 


$$
g(k)=\frac{d\left(U_{0}-W_{0}\right)-V}{d U_{0}}\left(\frac{V+d W_{0}}{d U_{0}}\right)^{k}, \quad k=0,1, \ldots, \infty .
$$

It can be verified that at stalling, $F=F_{s}=N_{f} \frac{k_{B} T}{d} \ln \hat{\rho}_{1}$, one gets $V=0$ and $g(0)=1-\hat{\rho}_{1}^{-1}$.

For comparison in the text, we need to compute $k_{a v}$ and $V(F)$ explicitly as follows:

1. $\mathbf{k}_{\mathbf{a v}}$ : Defining $\xi=\frac{V+d W_{0}}{d U_{0}}$ to simplify expressions, one gets from Eq. (C2),

$$
\begin{aligned}
g(k) & =(1-\xi) \xi^{k}, \\
k_{a v} & =\sum_{k=1}^{\infty} k g(k)=\frac{\xi}{1-\xi} .
\end{aligned}
$$

2. $\mathbf{V}(\mathbf{F})$ : Inserting $g(0)=\frac{d\left(U_{0}-W_{0}\right)-V}{d U_{0}}$ into Eq. (C1), we find for $V(F)$ a polynomial equation in $V$ to solve. Writing $v$ $=V / d W_{0}$, we find

$$
\begin{aligned}
\phi(v)= & \frac{\hat{\rho}_{1}}{N_{f}}\left[N_{f} \exp \left(-\frac{F d}{k_{B} T}\right)+\sum_{m=1}^{N_{f}-1}\left(1-\frac{1+v}{\hat{\rho}_{1}}\right)\left(N_{f}-m\right)\right. \\
& \left.\times \exp \left(-\frac{F d\left(N_{f}-m\right)}{N_{f} k_{B} T}\right)\right]-\frac{1}{N_{f}}\left[\left(1-\frac{1+v}{\hat{\rho}_{1}}\right)\right. \\
& \left.\times \sum_{m=1}^{N_{f}-1} m\left(\frac{1+v}{\hat{\rho}_{1}}\right)^{m-1}+N_{f}\left(\frac{1+v}{\hat{\rho}_{1}}\right)^{N_{f}-1}\right]-v=0 .
\end{aligned}
$$

Equation (C5) can be solved numerically using the Newton-Raphson method, for which the derivative of $\phi(v)$ with respect to $v$ is needed,

$$
\begin{aligned}
\phi^{\prime}(v)= & -\frac{\hat{\rho}_{1}}{N_{f}}\left[\sum_{m=1}^{N_{f}-1}\left(N_{f}-m\right)\left(N_{f}-m\right)\right. \\
& \left.\times \exp \left(-\frac{F d\left(N_{f}-m\right)}{N_{f} k_{B} T}\right)\right]+\frac{1}{N_{f} \hat{\rho}_{1}}\left[\sum_{m=1}^{N_{f}-1} m\left(\frac{1+v}{\hat{\rho}_{1}}\right)^{m-2}\right. \\
& \times\left(\frac{1+v}{\hat{\rho}_{1}}-\left(1-\frac{1+v}{\hat{\rho}_{1}}\right)(m-1)\right) \\
& \left.-N_{f}\left(N_{f}-1\right)\left(-\frac{1+v}{\hat{\rho}_{1}}\right)^{N_{f}-2}\right]-1 .
\end{aligned}
$$

The Newton-Raphson method requires a first guess value, say $v_{0}$, which can be taken as, e.g., Hill's value.

The solution of Démoulin et al.'s equation for a bundle of $N_{f}$ $=32$ filaments and supercritical density $\hat{\rho}_{1}=2.5$ is reported in Fig. 6, where it is compared with the results of our stochastic dynamics algorithm. The same is done by substituting $V(F)$ in Eqs. (C3) and (C4), for $g(0)$ and $k_{a v}$ in Figs. 4 and 5, respectively.

To predict within the present theory the value of $\tau^{O T}$, we need to compute the derivative of $V(F)$ with respect to $F$ at stalling, obtaining $\gamma^{D e m}$. From Eq. (C1), we get $d V / d F$ as an implicit function of $V(F)$ and $F$. At stalling $F=F_{s}, V\left(F_{s}\right)$ $=0$, we obtain

$$
\left.\frac{\partial V}{\partial F}\right|_{F=F_{s}}=-\frac{d^{2} W_{0}}{k_{B} T} \frac{\hat{\rho}_{1}^{1-N_{f}}\left[1+\sum_{m=1}^{N_{f}-1} \hat{\rho}_{1}^{m}\left(1-\hat{\rho}_{1}^{-1} \frac{\left(N_{f}-m\right)^{2}}{N_{f}^{2}}\right)\right]}{1+N_{f}^{-1} \sum_{m=1}^{N_{f}-1} m \hat{\rho}_{1}^{-m}+\left(N_{f}-1\right) \hat{\rho}_{1}^{-\left(N_{f}-1\right)}-N_{f}^{-1} \sum_{m=1}^{N_{f}-1} m \hat{\rho}_{1}^{-m}\left[1-\left(\hat{\rho}_{1}-1\right)(m-1)\right]} .
$$

For our conditions, the value is $\gamma^{D e m}=-(d V / d F)_{s}^{-1}=281.6$, which is in agreement with our results (see the main text).

${ }^{1}$ T. Risler, "Cytoskeleton and cell motility," in Encyclopedia of Complexity and Systems Science (Springer, 2009), pp. 1738-1774.

${ }^{2}$ S. Parekh, O. Chaudhuri, J. Theriot, and D. Fletcher, Nat. Cell Biol. 7, 1219 (2005).

${ }^{3}$ D. Smith and J. Liu, Phys. Biol. 10, 016004 (2013).

${ }^{4}$ C. Schreiber, M. Stewart, and T. Duke, Proc. Natl. Acad. Sci. U. S. A. 107, 9141 (2010).

${ }^{5}$ D. Démoulin, M. Carlier, J. Bibette, and J. Baudry, Proc. Natl. Acad. Sci. U. S. A. 111, 17845 (2014).

${ }^{6}$ M. Footer, J. Kerssemakers, J. Theriot, and M. Dogterom, Proc. Natl. Acad. Sci. U. S. A. 104, 2181 (2007).

${ }^{7}$ T. Hill, Proc. Natl. Acad. Sci. U. S. A. 78, 5613 (1981).

${ }^{8}$ T. Schaus and G. Borisy, Biophys. J. 95, 1393 (2008).

${ }^{9}$ G. Sander van Doorn, C. Tanase, B. Mulder, and M. Dogterom, Eur. Biophys. J. 29, 2 (2000).

${ }^{10}$ T. Hill and M. Kirschner, Int. Rev. Cytol. 78, 1 (1982).

${ }^{11}$ A. Perilli, C. Pierleoni, G. Ciccotti, and J.-P. Ryckaert, J. Chem. Phys. 144, 245102 (2016).

${ }^{12} \mathrm{M}$. Dogterom and B. Yurke, Science 278, 856 (1997).

${ }^{13}$ A. Mogilner and G. Oster, Eur. Biophys. J. 28, 235 (1999).
${ }^{14}$ K. Tsekouras, D. Lacoste, K. Mallick, and J.-F. Joanny, New J. Phys. 13, 103032 (2011).

${ }^{15}$ C. Peskin, G. Odell, and G. Oster, Biophys. J. 65, 316 (1993).

${ }^{16}$ R. Wang and A. Carlsson, New J. Phys. 16, 113047 (2014).

${ }^{17}$ A. Carlsson, Phys. Biol. 5, 036002 (2008).

${ }^{18} \mathrm{H}$. Wang, C. Peskin, and T. Elston, J. Theor. Biol. 221, 491 (2003).

${ }^{19}$ D. Gillespie, J. Phys. Chem. 81(25), 2340 (1977).

${ }^{20} \mathrm{P}$. Gaspard and E. Gerritsma, J. Theor. Biol. 247, 672 (2007).

${ }^{21}$ J. Norris, Markov Chains (Cambridge University Press, 1997).

${ }^{22}$ D. Gillespie, Markov Processes: An Introduction for Physical Scientists (Academic Press, Inc., San Diego, 1992).

${ }^{23}$ G. Kozyreff, "Brownian ratchet with a linear restoring force" (unpublished).

${ }^{24}$ P. Ranjith, D. Lacoste, K. Mallik, and J.-F. Joanny, Biophys. J. 96, 2146 (2009).

${ }^{25}$ J. Krawcyzk and J. Kiersfeld, Europhys. Lett. 93, 28006 (2011).

${ }^{26}$ D. Das, D. Das, and P. Ranjith, New J. Phys. 16, 063032 (2014).

${ }^{27}$ X. Li and A. Kolomeisky, J. Phys. Chem. B 119, 4653 (2015).

${ }^{28}$ A. Perilli, C. Pierleoni, and J.-P. Ryckaert, "Filament flexibility enhances power transduction of f-actin bundles" (unpublished). 